\title{
Robust exponential stability for uncertain stochastic neural networks with discrete and distributed time-varying delays
}

\author{
Hongyi $\mathrm{Li}^{\mathrm{a}}{ }^{\mathrm{a}, \mathrm{b}}$, Bing Chen ${ }^{\mathrm{b}, *}$, Qi Zhou ${ }^{\mathrm{a}, \mathrm{b}}$, Shengle Fang ${ }^{\mathrm{c}}$ \\ a Department of Mathematics, Bohai University, Jinzhou, Liaoning 121000, PR China \\ ${ }^{b}$ Institute of Complexity Science, Qingdao University, Qingdao 266071, PR China \\ c Institute of Nonlinear Complex Systems, China Three Gorges University, Yichang, Hubei 443002, PR China \\ Received 9 December 2007; received in revised form 22 January 2008; accepted 30 January 2008 \\ Available online 5 February 2008 \\ Communicated by A.R. Bishop
}

\begin{abstract}
This Letter deals with the problem of delay-dependent robust exponential stability in mean square for a class of uncertain stochastic Hopfield neural networks with discrete and distributed time-varying delays. Based on Lyapunov-Krasovskii functional and the stochastic stability theory, delay-dependent stability criteria are obtained in terms of linear matrix inequalities (LMIs). Because of introducing some free-weighting matrices to develop the stability criteria, the proposed stability conditions have less conservatism. Numerical examples are given to illustrate the effectiveness of our results.
\end{abstract}

(C) 2008 Elsevier B.V. All rights reserved.

Keywords: Neural networks; Exponential stability; Stochastic systems; Uncertain systems; LMIs

\section{Introduction}

Over last decades, the study of Hopfield neural networks has received considerable attention since they play an important role in various fields such as signal processing, image processing, pattern recognition and optimization problems [1]. As well known, in practice time-delays are often encountered in various engineering, biological, and economical systems. Due to the finite speed of information processing, the existence of time-delays frequently causes oscillation, divergence, or even instability in neural networks. Therefore, the stability analysis for Hopfield neural networks with time-delay has attracted a large amount of research interest and many sufficient conditions have been proposed to guarantee the stability of neural networks with various type of time delays, see for example [2-15], and the references therein.

On the other hand, in hardware implementation of neural networks, stochastic disturbances are nearly inevitable owing to thermal noise in electronic devices. In [16], it has been shown that certain stochastic inputs could destabilize a neural network. Therefore, increasing attention has been paid to the problem of stochastic stability for stochastic time-delay neural networks [17-32]. In [24], the problem of stochastic robust stability of uncertain stochastic Hopfield neural networks with time-varying delays was investigated. Recently, by introducing slack matrices, delay-dependent robust stability criteria for uncertain stochastic neural networks with time-varying delay were obtained in $[25,26]$. As discussed in $[2,3,27-30]$, distributed delays should be incorporated into the model due to the fact that there may exist a distribution of propagation delays over a period of time in some cases. Recently, some results on stability of stochastic neural networks with finite distributed delay have been presented in [28-30]. To the best of our

\footnotetext{
This work is partially supported by the Natural Science Foundation of China (60674055, 60774047), the Taishan Scholar programme of Shandong Province.

* Corresponding author. Tel.: +86 532 86953607; fax: +86 53285953607.

E-mail address: chenbing1958@yahoo.com.cn (B. Chen).
} 
knowledge, so far, no result on the delay-dependent robust exponential stability analysis for uncertain stochastic neural networks with discrete and distributed time-varying delays is available in the literature.

Motivated by the above observation, in this Letter, we investigate the problem of delay-dependent robust exponential stability for a class of uncertain stochastic neural networks with discrete and distributed time-varying delays. The uncertainties are assumed to be norm-bounded. Based on Lyapunov-Krasovskii stability theory and free-weighting matrix method, new delay-dependent robust exponential stability criteria are presented in terms of LMIs. Finally, some numerical examples are given to illustrate the effectiveness of the developed methods.

Notation. Through this Letter, $\mathbb{R}^{n}$ and $\mathbb{R}^{n \times m}$ denote the $n$-dimensional Euclidean space and the set of all $n \times m$ real matrices, respectively. $I$ is the identity matrix with appropriate dimensions; $M^{T}$ stands for the transpose of the matrix $M$; For symmetric matrices $X$ and $Y$, the notation $X>Y$ (respectively $X \geqslant Y$ ) means that the $X-Y$ is positive definite (respectively, positive semidefinite) $|\cdot|$ refers the Euclidean vector norm, the notation $\operatorname{diag}\left\{G_{1}, G_{2}, \ldots, G_{n}\right\}$ denotes a block-diagonal matrix with diagonal blacks being the matrices $G_{1}, G_{2}, \ldots, G_{n} .\left(\Omega, \mathcal{F},\left\{\mathcal{F}_{t}\right\}_{t} \geqslant 0, P\right)$ is a probability space with a filtration $\left\{\mathcal{F}_{t}\right\}_{t} \geqslant 0$ satisfying the usual conditions (i.e. the filtration contains all $P$-null sets and is right continuous), $L_{\mathcal{F}_{0}}^{2}\left([-2 \bar{\tau}, 0] ; \mathbb{R}^{n}\right)$ stands for the family of all $\mathcal{F}_{0}$ measurable $C\left([-2 \bar{\tau}, 0] ; \mathbb{R}^{n}\right)$-valued random variable $\xi=\{\xi(\theta):-2 \bar{\tau} \leqslant \theta \leqslant 0\}$ such that $\sup _{-2 \bar{\tau} \leqslant \theta \leqslant 0} \mathcal{E}|\xi(\theta)|<\infty$, where $\mathcal{E}(\cdot)$ is the mathematical expectation; " $*$ " denotes a block that is readily inferred by symmetry. Matrices, if not explicitly stated, are assumed to have compatible dimensions.

\section{Problem formulation}

The Hopfield neural network with both discrete and distributed delays can be described as [28] by the following model:

$$
\dot{y}_{i}(t)=-a_{i} y_{i}(t)+\sum_{j=1}^{n} w_{i j}^{0} g_{j}\left(y_{j}(t)\right)+\sum_{j=1}^{n} w_{i j}^{1} g_{j}\left(y_{j}(t-h(t))\right)+\sum_{j=1}^{n} e_{i j}^{1} \int_{t-r(t)}^{t} g_{j}\left(y_{j}(s)\right) \mathrm{d} s+I_{i}, \quad i=1,2, \ldots, n,
$$

or equivalently the vector form

$$
\dot{y}(t)=-A y(t)+W_{0} g(y(t))+W_{1} g(y(t-h(t)))+E_{1} \int_{t-r(t)}^{t} g(y(s)) \mathrm{d} s+I,
$$

where $y(t)=\left[y_{1}(t), y_{2}(t), \ldots, y_{n}(t)\right]^{T} \in \mathbb{R}^{n}$ denotes the state vector associated with $n$ neurons. The matrix $A=\operatorname{diag}\left(a_{1}, a_{2}\right.$, $\left.\ldots, a_{n}\right)$ is a diagonal matrix with positive entries $a_{i}>0 . W_{0}=\left(w_{i j}^{0}\right)_{n \times n}, W_{1}=\left(w_{i j}^{1}\right)_{n \times n}$ and $E_{1}=\left(e_{i j}^{1}\right)_{n \times n}$ are the connection weights, the discretely delayed connection weights, and the distributively delayed connection weights, of the $j$ th neuron on the $i$ the neuron, respectively. $g(x)=\left[g_{1}\left(x_{1}\right), g_{2}\left(x_{2}\right), \ldots, g_{n}\left(x_{n}\right)\right]^{T} \in \mathbb{R}^{n}$ is the activation function with $g(0)=0 . I=\left[I_{1}, I_{2}, \ldots, I_{n}\right]$ is a constant external input. The bounded function $h(t)$ and $r(t)$ represent unknown discrete and distributed delays of systems with $0<h(t) \leqslant \bar{h}, \dot{h}(t) \leqslant \mu$ and $0<r(t) \leqslant \bar{r}$.

(H1). The activation function $g$ is bounded and satisfies Lipschitz condition

$$
\left|g\left(x_{1}\right)-g\left(x_{2}\right)\right| \leqslant L\left|x_{1}-x_{2}\right|, \quad \forall x_{1}, x_{2} \in \mathbb{R},
$$

where $L=\operatorname{diag}\left(l_{1}, l_{2}, \ldots, l_{n}\right)>0$ is a positive diagonal matrix. Then, by (H1) we can have

$$
|g(x)| \leqslant L|x|, \quad \forall x \in \mathbb{R} .
$$

As above mentioned, it is reasonable to assume that the neural network (2) has only one equilibrium point $y^{*}=\left(y_{1}^{*}, y_{2}^{*}, \ldots, y_{n}^{*}\right)$. Then, we will shift the equilibrium point $y^{*}$ to the origin. The transformation $x(\cdot)=y(\cdot)-y^{*}$ puts system (2) into the following form:

$$
\dot{x}(t)=-A x(t)+W_{0} f(x(t))+W_{1} f(x(t-h(t)))+E_{1} \int_{t-r(t)}^{t} f(x(s)) \mathrm{d} s,
$$

where $x(t)$ is the state vector of the transformation system, $f_{j}(x(t))=g_{j}\left(y_{j}(t)+y_{j}^{*}\right)-g_{j}\left(y_{j}^{*}\right)$ with $f_{j}(x(0))=0$ for $j=$ $1,2, \ldots, n$. Then, from (3), we have,

$$
f^{T}(x) f(x) \leqslant x^{T}(t) L^{T} \operatorname{Lx}(t)
$$

As above discussed, in reality, it is often the case that the connection weights of the neurons include uncertainties, and the neural network is disturbed by environmental noises that affect the stability of the equilibrium. In this Letter, as in [28-30], the Hopfield 
neural network with parameter uncertainties and stochastic perturbations can be described as follows:

$$
\begin{aligned}
\mathrm{d} x(t)= & {\left[-A(t) x(t)+W_{0}(t) f(x(t))+W_{1}(t) f(x(t-h(t)))+E_{1} \int_{t-r(t)}^{t} f(x(s)) \mathrm{d} s\right] \mathrm{d} t } \\
& +\left[C(t) x(t)+D(t) x(t-h(t))+W_{2}(t) f(x(t))+W_{3}(t) f(x(t-h(t)))+E_{2} \int_{t-r(t)}^{t} f(x(s)) \mathrm{d} s\right] \mathrm{d} \omega(t), \\
x(t)= & \phi(t), \quad \forall t \in[-2 \bar{\tau}, 0], \bar{\tau}=\max \{\bar{h}, \bar{r}\}
\end{aligned}
$$

where $\omega(t)$ denotes a one-dimensional Brownian motion satisfying $\mathcal{E}\{\mathrm{d} \omega(t)\}=0$ and $\mathcal{E}\left\{\mathrm{d} \omega(t)^{2}\right\}=\mathrm{d} t$. The matrices $A(t)=A+$ $\Delta A(t), W_{0}(t)=W_{0}+\Delta W_{0}(t), W_{1}(t)=W_{1}+\Delta W_{1}(t), C(t)=C+\Delta C(t), D(t)=D+\Delta D(t), W_{2}(t)=W_{2}+\Delta W_{2}(t)$ and $W_{3}(t)=W_{3}+\Delta W_{3}(t)$, where $A=\operatorname{diag}\left(a_{1}, a_{2}, \ldots, a_{n}\right)$ has positive entries $a_{i}>0, W_{0}, W_{1}, W_{2}$ and $W_{3}$ are connection weight matrices with appropriate dimensions. In this system, the parameter uncertainties are assumed to be of the following form:

$$
\left[\Delta A(t), \Delta W_{0}(t), \Delta W_{1}(t), \Delta C(t), \Delta D(t), \Delta W_{2}(t), \Delta W_{3}(t)\right]=H F(t)\left[T_{1}, T_{2}, T_{3}, T_{4}, T_{5}, T_{6}, T_{7}\right]
$$

where $\Delta A(t)$ is a diagonal matrix, $H$ and $T_{i}(i=1, \ldots, 7)$ are known real constant matrices of appropriate dimensions. The matrix $F(t)$, which may be time-varying, is unknown and satisfies

$$
F^{T}(t) F(t) \leqslant I
$$

It is assumed that all the elements of $F(t)$ are Lebesgue measurable. $\Delta A(t), \Delta W_{0}(t), \Delta W_{1}(t), \Delta C(t), \Delta D(t), \Delta W_{2}(t)$ and $\Delta W_{3}(t)$ are said to be admissible if both (8) and (9) hold. $\phi(t) \in C\left([-2 \bar{\tau}, 0] ; \mathbb{R}^{n}\right)$ is the initial function. $f(x)=$ $\left[f_{1}\left(x_{1}\right), f_{2}\left(x_{2}\right), \ldots, f_{n}\left(x_{n}\right)\right]^{T} \in \mathbb{R}^{n}$ is the activation function with $f(0)=0$. Before formulating the problem considered in this Letter, we first introduce the following concept of robust exponential stability in mean square for the system (6)-(7).

Definition 1. The trivial solution of the uncertain stochastic Hopfield neural network (6) is said to be robustly exponentially stable in mean square, if there exist positive scalars $\alpha$ and $\beta$, such that

$$
\mathcal{E}\left\{\|x(t, \phi)\|^{2}\right\} \leqslant \alpha e^{-\beta t} \mathcal{E}\left\{\sup _{-2 \bar{\tau} \leqslant s \leqslant 0}\|\phi(s)\|^{2}\right\}
$$

for all admissible uncertainties satisfying (8)-(9).

The following lemmas will be used in the proofs of our results.

Lemma 1 (Schur complement). Given constant $\Omega_{1}, \Omega_{2}$ and $\Omega_{3}$ with appropriate dimensions, where $\Omega_{1}^{T}=\Omega_{1}$ and $\Omega_{2}^{T}=\Omega_{2}>0$, then

$$
\Omega_{1}+\Omega_{3}^{T} \Omega_{2}^{-1} \Omega_{3}<0
$$

if and only if

$$
\left[\begin{array}{cc}
\Omega_{1} & \Omega_{3}^{T} \\
* & -\Omega^{2}
\end{array}\right]<0 \quad \text { or } \quad\left[\begin{array}{cc}
-\Omega_{2} & \Omega_{3} \\
* & \Omega_{1}
\end{array}\right]<0
$$

Lemma 2. (See Ref. [33].) For any constant matrix $M>0$, any scalars a and $b$ with $a<b$, and a vector function $x(t):[a, b] \rightarrow \mathbb{R}^{n}$ such that the integrals concerned as well defined, then the following holds

$$
\left[\int_{a}^{b} x(s) \mathrm{d} s\right]^{T} M\left[\int_{a}^{b} x(s) \mathrm{d} s\right] \leqslant(b-a) \int_{a}^{b} x^{T}(s) M x(s) \mathrm{d} s .
$$

Lemma 3. (See Ref. [34].) Let $M, E$ and $F(t)$ be real matrices of appropriate dimensions with $F(t)$ satisfying $F^{T}(t) F(t) \leqslant I$. Then, the following inequality holds for any $\varepsilon>0$,

$$
M F(t) E+E^{T} F^{T}(t) M^{T} \leqslant \varepsilon M M^{T}+\varepsilon^{-1} E^{T} E .
$$




\section{New stability results}

Defining two new state variables for the stochastic neural network (6),

$$
\begin{aligned}
& y(t)=-A(t) x(t)+W_{0}(t) f(x(t))+W_{1}(t) f(x(t-h(t)))+E_{1} \int_{t-r(t)}^{t} f(x(s)) \mathrm{d} s, \\
& g(t)=C(t) x(t)+D(t) x(t-h(t))+W_{2}(t) f(x(t))+W_{3}(t) f(x(t-h(t)))+E_{2} \int_{t-r(t)}^{t} f(x(s)) \mathrm{d} s .
\end{aligned}
$$

Then, the stochastic neural network (6) can be represented as

$$
\mathrm{d} x(t)=y(t) \mathrm{d} t+g(t) \mathrm{d} \omega(t) .
$$

Moreover, the following equality holds,

$$
x(t)-x(t-h(t))=\int_{t-h(t)}^{t} \mathrm{~d} x(s)=\int_{t-h(t)}^{t} y(s) \mathrm{d} s+\int_{t-h(t)}^{t} g(s) \mathrm{d} \omega(s) .
$$

Theorem 1. Given scalars $\bar{h}>0, \mu$ and $\bar{r}>0$. The equilibrium solution of stochastic neural network (6) is robustly exponentially stable in mean square if there exist matrices $P>0, Q>0, R>0, Z>0, M_{k}, N_{k}$ and $S_{k}(k=1, \ldots, 6)$ as well as constants $b_{1}>0, b_{2}>0, \varepsilon_{1}>0$ and $\varepsilon_{2}>0$, such that the following LMI holds:

$$
\left[\begin{array}{ccccc}
\Xi_{11} & -N & M E_{1}+S E_{2} & M H & S H \\
* & -\frac{1}{\bar{h}} R & 0 & 0 & 0 \\
* & * & -\frac{1}{\bar{r}} Z & 0 & 0 \\
* & * & * & -\varepsilon_{1} I & 0 \\
* & * & * & * & -\varepsilon_{2} I
\end{array}\right]<0,
$$

where

$$
\Xi_{11}=\left(\phi_{i j}\right)_{6 \times 6}+\varepsilon_{1} \bar{T}_{1}^{T} \bar{T}_{1}+\varepsilon_{2} \bar{T}_{2}^{T} \bar{T}_{2}
$$

with

$$
\begin{aligned}
& \phi_{11}=Q+N_{1}+N_{1}^{T}-M_{1} A-A^{T} M_{1}^{T}+S_{1} C+C^{T} S_{1}^{T}+b_{1} L^{T} L, \\
& \phi_{12}=-N_{1}+N_{2}^{T}-A^{T} M_{2}^{T}+S_{1} D+C^{T} S_{2}^{T}, \quad \phi_{13}=P+N_{3}^{T}-M_{1}-A^{T} M_{3}^{T}+C^{T} S_{3}^{T}, \\
& \phi_{14}=N_{4}^{T}-S_{1}-A^{T} M_{4}^{T}+C^{T} S_{4}^{T}, \quad \phi_{15}=N_{5}^{T}+M_{1} W_{0}-A^{T} M_{5}^{T}+S_{1} W_{2}+C^{T} S_{5}^{T}, \\
& \phi_{16}=N_{6}^{T}+M_{1} W_{1}-A^{T} M_{6}^{T}+S_{1} W_{3}+C^{T} S_{6}^{T}, \quad \phi_{23}=-N_{3}^{T}-M_{2}+D^{T} S_{3}^{T}, \\
& \phi_{22}=-(1-\mu) Q-N_{2}-N_{2}^{T}+S_{2} D+D^{T} S_{2}^{T}+b_{2} L^{T} L, \quad \phi_{24}=-N_{4}^{T}-S_{2}+D^{T} S_{4}^{T}, \\
& \phi_{25}=-N_{5}^{T}+M_{2} W_{0}+S_{2} W_{2}+D^{T} S_{5}^{T}, \quad \phi_{26}=-N_{6}^{T}+M_{2} W_{1}+S_{2} W_{3}+D^{T} S_{6}^{T}, \\
& \phi_{33}=\bar{h} R-M_{3}-M_{3}^{T}, \quad \phi_{34}=-M_{4}^{T}-S_{3}, \quad \phi_{35}=M_{3} W_{0}-M_{5}^{T}+S_{3} W_{2}, \\
& \phi_{36}=M_{3} W_{1}-M_{6}^{T}+S_{3} W_{3}, \quad \phi_{44}=P-S_{4}-S_{4}^{T}, \quad \phi_{45}=M_{4} W_{0}-S_{5}^{T}+S_{4} W_{2}, \\
& \phi_{46}=M_{4} W_{1}-S_{6}^{T}+S_{4} W_{3}, \quad \phi_{55}=-b_{1} I+\bar{r} Z+M_{5} W_{0}+W_{0}^{T} M_{5}^{T}+S_{5} W_{2}+W_{2}^{T} S_{5}^{T}, \\
& \phi_{56}=M_{5} W_{1}+W_{0}^{T} M_{6}^{T}+S_{5} W_{3}+W_{2}^{T} S_{6}^{T}, \quad \phi_{66}=-b_{2} I+M_{6} W_{1}+W_{1}^{T} M_{6}^{T}+S_{6} W_{3}+W_{3}^{T} S_{6}^{T}, \\
& \bar{T}_{1}=\left[-T_{1}, 0,0,0, T_{2}, T_{3}\right], \quad \bar{T}_{2}=\left[T_{4}, T_{5}, 0,0, T_{6}, T_{7}\right], \quad N=\left[N_{1}^{T}, N_{2}^{T}, N_{3}^{T}, N_{4}^{T}, N_{5}^{T}, N_{6}^{T}\right]^{T}, \\
& M=\left[M_{1}^{T}, M_{2}^{T}, M_{3}^{T}, M_{4}^{T}, M_{5}^{T}, M_{6}^{T}\right]^{T}, \quad S=\left[S_{1}^{T}, S_{2}^{T}, S_{3}^{T}, S_{4}^{T}, S_{5}^{T}, S_{6}^{T}\right]^{T} .
\end{aligned}
$$

Proof. Consider the Lyapunov-Krasovskii functional as follows:

$$
V\left(x_{t}, t\right)=x^{T}(t) P x(t)+\int_{t-h(t)}^{t} x^{T}(s) Q x(s) \mathrm{d} s+\int_{-\bar{h}}^{0} \int_{t+\theta}^{t} y^{T}(s) R y(s) \mathrm{d} s \mathrm{~d} \theta+\int_{-\bar{r}}^{0} \int_{t+\theta}^{t} f^{T}(x(s)) Z f(x(s)) \mathrm{d} s \mathrm{~d} \theta,
$$


where $x_{t}=\{x(t+\theta):-2 \bar{\tau} \leqslant \theta \leqslant 0\}$. Then, it can be obtained by Itô's differential formula that

$$
\mathrm{d} V\left(x_{t}, t\right)=\mathcal{L} V\left(x_{t}, t\right) \mathrm{d} t+2 x^{T}(t) P g(t) \mathrm{d} \omega(t),
$$

where

$$
\begin{aligned}
\mathcal{L} V\left(x_{t}, t\right)= & 2 x^{T}(t) P y(t)+g^{T}(t) P g(t)+x^{T}(t) Q x(t)-(1-\dot{h}(t)) x^{T}(t-h(t)) Q x(t-h(t)) \\
& +\bar{r} f^{T}(x(t)) Z f(x(t))+\bar{h} y^{T}(t) R y(t)-\int_{t-\bar{h}}^{t} y^{T}(s) R y(s) \mathrm{d} s-\int_{t-\bar{r}}^{t} f^{T}(x(s)) Z f(x(s)) \mathrm{d} s \\
\leqslant & x^{T}(t) Q x(t)+2 x^{T}(t) P y(t)-(1-\mu) x^{T}(t-h(t)) Q x(t-h(t))+\bar{r} f^{T}(x(t)) Z f(x(t)) \\
& +g^{T}(t) P g(t)+\bar{h} y^{T}(t) R y(t)-\int_{t-h(t)}^{t} y^{T}(s) R y(s) \mathrm{d} s-\int_{t-r(t)}^{t} f^{T}(x(s)) Z f(x(s)) \mathrm{d} s .
\end{aligned}
$$

Then, according to Lemma 2 and using $0<h(t) \leqslant \bar{h}$ and $0<r(t) \leqslant \bar{r}$, we have

$$
-\int_{t-h(t)}^{t} y^{T}(s) R y(s) \mathrm{d} s \leqslant-\frac{1}{\bar{h}}\left[\int_{t-h(t)}^{t} y(s) \mathrm{d} s\right]^{T} R\left[\int_{t-h(t)}^{t} y(s) \mathrm{d} s\right],
$$

and

$$
-\int_{t-r(t)}^{t} f^{T}(x(s)) Z f(x(s)) \mathrm{d} s \leqslant-\frac{1}{\bar{r}}\left[\int_{t-r(t)}^{t} f(x(s)) \mathrm{d} s\right]^{T} Z\left[\int_{t-r(t)}^{t} f(x(s)) \mathrm{d} s\right] .
$$

Now, define new vector $e(t)$ as

$$
e^{T}(t)=\left[x^{T}(t), x^{T}(t-h(t)), y^{T}(t), g^{T}(t), f^{T}(x(t)), f^{T}(x(t-h(t)))\right] .
$$

From (10), (11) and (13), we can see that the following equations are true for any matrices $N, M$ and $S$ with appropriate dimensions.

$$
\begin{aligned}
& \eta_{1}(t)=2 e^{T}(t) N\left[x(t)-x(t-h(t))-\int_{t-h(t)}^{t} y(s) \mathrm{d} s-\int_{t-h(t)}^{t} g(s) \mathrm{d} \omega(s)\right], \\
& \eta_{2}(t)=2 e^{T}(t) M\left[-A(t) x(t)+W_{0}(t) f(x(t))+W_{1}(t) f(x(t-h(t)))+E_{1} \int_{t-r(t)}^{t} f(x(s)) \mathrm{d} s-y(t)\right], \\
& \eta_{3}(t)=2 e^{T}(t) S\left[C(t) x(t)+D(t) x(t-h(t))+W_{2}(t) f(x(t))+W_{3}(t) f(x(t-h(t)))+E_{2} \int_{t-r(t)}^{t} f(x(s)) \mathrm{d} s-g(t)\right] .
\end{aligned}
$$

It is obvious from (5) that

$$
\begin{aligned}
& -b_{1} f^{T}(x(t)) f(x(t))+b_{1} x^{T}(t) L^{T} L x(t) \geqslant 0, \\
& -b_{2} f^{T}(x(t-h(t))) f(x(t-h(t)))+b_{2} x^{T}(t-h(t)) L^{T} L x(t-h(t)) \geqslant 0 .
\end{aligned}
$$

Then, substituting (17)-(18) into (16), and then adding $\eta_{1}(t), \eta_{2}(t), \eta_{3}(t),(22)$ and (23) to the right side of (16) produce that

$$
\mathrm{d} V\left(x_{t}, t\right) \leqslant \zeta^{T}(t) \Phi \zeta(t)+\xi(\mathrm{d} \omega(t))
$$

where

$$
\Phi=\left[\begin{array}{ccc}
\bar{\Omega} & -N & M E_{1}+S E_{2} \\
* & -\frac{1}{\bar{\tau}} R & 0 \\
* & * & -\frac{1}{\bar{r}} Z
\end{array}\right]
$$

with 


$$
\begin{aligned}
& \bar{\Omega}=\left(\phi_{i j}\right)_{6 \times 6}+\Delta \Omega_{1}+\Delta \Omega_{2}, \\
& \Delta \Omega_{1}=M H F(t) \bar{T}_{1}+\bar{T}_{1}^{T} F^{T}(t) H^{T} M^{T}, \\
& \Delta \Omega_{2}=S H F(t) \bar{T}_{2}+\bar{T}_{2}^{T} F^{T}(t) H^{T} S^{T}, \\
& \zeta^{T}(t)=\left[e^{T}(t), \int_{t-h(t)}^{t} y^{T}(s) \mathrm{d} s, \int_{t-r(t)}^{t} f^{T}(x(s)) \mathrm{d} s\right], \\
& \xi(\mathrm{d} \omega(t))=-2 e^{T}(t) N \int_{t-h(t)}^{t} g(s) \mathrm{d} \omega(s)+2 x^{T}(t) P g(t) \mathrm{d} \omega(t) .
\end{aligned}
$$

According to (8) and Lemma $3, \Delta \Omega_{1}$ and $\Delta \Omega_{2}$ satisfy the following inequalities:

$$
\Delta \Omega_{1} \leqslant \varepsilon_{1}^{-1} M H H^{T} M^{T}+\varepsilon_{1} \bar{T}_{1}^{T} \bar{T}_{1}, \quad \Delta \Omega_{2} \leqslant \varepsilon_{2}^{-1} S H H^{T} S^{T}+\varepsilon_{2} \bar{T}_{2}^{T} \bar{T}_{2} .
$$

From applying Schur complement to (14), we can obtain

$$
\mathrm{d} V\left(x_{t}, t\right) \leqslant-a\left(\|x(t)\|^{2}+\|x(t-h(t))\|^{2}+\|x(t-r(t))\|^{2}\right) \mathrm{d} t+\xi(\mathrm{d} \omega(t)),
$$

where $a=\lambda_{\min }\{-\Phi\}>0$. From the definitions of $V\left(x_{t}, t\right), y(t)$ and $f(x(t))$, there exist positive scalars $\delta_{1}, \delta_{2}, \delta_{3}$ and $\delta_{4}$ such that the following inequality holds

$$
V\left(x_{t}, t\right) \leqslant \delta_{1}\|x(t)\|^{2}+\delta_{2} \int_{t-\bar{\tau}}^{t}\|x(\alpha)\|^{2} \mathrm{~d} \alpha+\delta_{3} \int_{t-\bar{\tau}}^{t}\|x(\alpha-h(\alpha))\|^{2} \mathrm{~d} \alpha+\delta_{4} \int_{t-\bar{\tau}}^{t}\|x(\alpha-r(\alpha))\|^{2} \mathrm{~d} \alpha .
$$

Choose $\beta>0$, such that

$$
\beta \max \left\{\delta_{1}+\delta_{2} \bar{\tau} e^{\beta \bar{\tau}}, \delta_{3} \bar{\tau} e^{\beta \bar{\tau}}, \delta_{4} \bar{\tau} e^{\beta \bar{\tau}}\right\} \leqslant a .
$$

Therefore,

$$
\begin{aligned}
\mathrm{d}\left[e^{\beta t} V\left(x_{t}, t\right)\right] \leqslant & e^{\beta t}\left[\left(\beta \delta_{1}-a\right)\|x(t)\|^{2}-a\|x(t-h(t))\|^{2}-a\|x(t-r(t))\|^{2}+\beta \delta_{2} \int_{t-\bar{\tau}}^{t}\|x(\alpha)\|^{2} \mathrm{~d} \alpha\right. \\
& \left.+\beta \delta_{3} \int_{t-\bar{\tau}}^{t}\|x(\alpha-h(\alpha))\|^{2} \mathrm{~d} \alpha+\beta \delta_{4} \int_{t-\bar{\tau}}^{t}\|x(\alpha-r(\alpha))\|^{2} \mathrm{~d} \alpha\right] \mathrm{d} t+e^{\beta t} \xi(\mathrm{d} \omega(t)) .
\end{aligned}
$$

Thus, integrating both sides of the above inequality from 0 to $T>0$ and then taking expectation show that

$$
\begin{aligned}
\mathcal{E}\left(e^{\beta t} V\left(x_{T}, T\right)\right) \leqslant & J_{1}+\left(\beta \delta_{1}-a\right) \mathcal{E}\left\{\int_{0}^{T} e^{\beta t}\|x(t)\|^{2} \mathrm{~d} t\right\}-a \mathcal{E}\left\{\int_{0}^{T} e^{\beta t}\|x(t-h(t))\|^{2} \mathrm{~d} t\right\}-a \mathcal{E}\left\{\int_{0}^{T} e^{\beta t}\|x(t-r(t))\|^{2} \mathrm{~d} t\right\} \\
& +\beta \delta_{2} \mathcal{E}\left\{\int_{0}^{T} \int_{t-\bar{\tau}}^{t} e^{\beta t}\|x(\alpha)\|^{2} \mathrm{~d} \alpha \mathrm{d} t\right\}+\beta \delta_{3} \mathcal{E}\left\{\int_{0}^{T} \int_{t-\bar{\tau}}^{t} e^{\beta t}\|x(\alpha-h(\alpha))\|^{2} \mathrm{~d} \alpha \mathrm{d} t\right\} \\
& +\beta \delta_{4} \mathcal{E}\left\{\int_{0}^{T} \int_{t-\bar{\tau}}^{t} e^{\beta t}\|x(\alpha-r(\alpha))\|^{2} \mathrm{~d} \alpha \mathrm{d} t\right\},
\end{aligned}
$$

where

$$
J_{1}=\left[\delta_{1}+2 \bar{\tau} \delta_{2}+2 \bar{\tau} \delta_{3}+2 \bar{\tau} \delta_{4}\right] \sup _{-2 \bar{\tau} \leqslant s \leqslant 0} \mathcal{E}\|\phi(s)\|^{2} .
$$

Consequently, by changing the integration sequence, the following inequalities hold, 


$$
\begin{aligned}
& \int_{0}^{T} \int_{t-\bar{\tau}}^{t} e^{\beta t}\|x(\alpha)\|^{2} \mathrm{~d} \alpha \mathrm{d} t \leqslant \int_{-\bar{\tau}}^{T}\left(\int_{\alpha \vee 0}^{(\alpha+\bar{\tau}) \wedge T} e^{\beta t} d t\right)\|x(\alpha)\|^{2} \mathrm{~d} \alpha \leqslant \int_{-\bar{\tau}}^{T} \bar{\tau} e^{\beta(\alpha+\bar{\tau})}\|x(\alpha)\|^{2} \mathrm{~d} \alpha \\
& \leqslant \bar{\tau} e^{\beta \bar{\tau}} \int_{0}^{T} e^{\beta t}\|x(t)\|^{2} \mathrm{~d} t+\bar{\tau} e^{\beta \bar{\tau}} \int_{-\bar{\tau}}^{0}\|\phi(s)\|^{2} \mathrm{~d} s \\
& \leqslant \bar{\tau} e^{\beta \bar{\tau}} \int_{0}^{T} e^{\beta t}\|x(t)\|^{2} \mathrm{~d} t+2 \bar{\tau}^{2} e^{\beta \bar{\tau}} \sup _{-2 \bar{\tau} \leqslant s \leqslant 0}\|\phi(s)\|^{2}, \\
& \int_{0}^{T} \int_{t-\bar{\tau}}^{t} e^{\beta t}\|x(\alpha-h(\alpha))\|^{2} \mathrm{~d} \alpha \mathrm{d} t \leqslant \bar{\tau} e^{\beta \bar{\tau}} \int_{0}^{T} e^{\beta t}\|x(t-h(t))\|^{2} \mathrm{~d} t+2 \bar{\tau}^{2} e^{\beta \bar{\tau}} \sup _{-2 \bar{\tau} \leqslant s \leqslant 0}\|\phi(s)\|^{2}, \\
& \int_{0}^{T} \int_{t-\bar{\tau}}^{t} e^{\beta t}\|x(\alpha-r(\alpha))\|^{2} \mathrm{~d} \alpha \mathrm{d} t \leqslant \bar{\tau} e^{\beta \bar{\tau}} \int_{0}^{T} e^{\beta t}\|x(t-r(t))\|^{2} \mathrm{~d} t+2 \bar{\tau}^{2} e^{\beta \bar{\tau}} \sup _{-2 \bar{\tau} \leqslant s \leqslant 0}\|\phi(s)\|^{2} .
\end{aligned}
$$

After substituting (29)-(31) into the right side of (28), and the using (27), we can obtain

$$
\mathcal{E}\left(e^{\beta t} V\left(x_{T}, T\right)\right) \leqslant J_{1}+J_{2},
$$

where

$$
J_{2}=\left(2 \beta \delta_{2} \bar{\tau}^{2} e^{\beta \bar{\tau}}+2 \beta \delta_{3} \bar{\tau}^{2} e^{\beta \bar{\tau}}+2 \beta \delta_{4} \bar{\tau}^{2} e^{\beta \bar{\tau}}\right) \sup _{-2 \bar{\tau} \leqslant s \leqslant 0} \mathcal{E}\|\phi(s)\|^{2} .
$$

So,

$$
\mathcal{E}\left\{\|x(T, \phi)\|^{2}\right\} \leqslant \frac{J_{1}+J_{2}}{\lambda_{\min }(P)} e^{-\beta t},
$$

then it can be shown that for any $T>0$,

$$
\mathcal{E}\left\{\|x(T, \phi)\|^{2}\right\} \leqslant \alpha e^{-\beta t} \sup _{-2 \bar{\tau} \leqslant s \leqslant 0} \mathcal{E}\|\phi(s)\|^{2},
$$

where

$$
\alpha=\frac{1}{\lambda_{\min }(P)}\left[\delta_{1}+2 \bar{\tau} \delta_{2}+2 \bar{\tau} \delta_{3}+2 \bar{\tau} \delta_{4}+2 \beta \delta_{2} \bar{\tau}^{2} e^{\beta \tau}+2 \beta \delta_{3} \bar{\tau}^{2} e^{\beta \bar{\tau}}+2 \beta \delta_{4} \bar{\tau}^{2} e^{\beta \bar{\tau}}\right] .
$$

Consequently, by the proof of Lyapunov stability theory in [16], and Definition 1, we know that the equilibrium solution of the stochastic neural network (6) is robustly exponentially stochastically stable in mean square for any $h(t), r(t)$ satisfying $0<h(t) \leqslant \bar{h}$, $\dot{h}(t) \leqslant \mu$ and $0<r(t) \leqslant \bar{r}$. The proof is thus completed.

Remark 1. The results of Theorem 1 can be easily extended to the following uncertain stochastic neural networks with multiple discrete and distributed delays,

$$
\begin{aligned}
\mathrm{d} x(t)= & {\left[-A(t) x(t)+W_{0}(t) f(x(t))+\sum_{i=1}^{n} W_{1 i}(t) f\left(x\left(t-h_{i}(t)\right)\right)+\sum_{i=1}^{n} E_{1 i} \int_{t-r_{i}(t)}^{t} f(x(s)) \mathrm{d} s\right] \mathrm{d} t } \\
& +\left[C(t) x(t)+\sum_{i=1}^{n} D_{i}(t) x\left(t-h_{i}(t)\right)+W_{2}(t) f(x(t))+\sum_{i=1}^{n} W_{3 i}(t) f\left(x\left(t-h_{i}(t)\right)\right)\right. \\
& \left.+\sum_{i=1}^{n} E_{2 i} \int_{t-r_{i}(t)}^{t} f(x(s)) d s\right] \mathrm{d} \omega(t),
\end{aligned}
$$

by following similar lines as in the proof of Theorem 1. In order to avoid complicated notations, here we only consider the robust stability of system (6). 
Remark 2. When the derivative of $h(t)$ is unknown, and the delay $h(t)$ satisfies $0<h(t) \leqslant \bar{h}$, by setting $Q=0$ in (15), we can know that the system (6) is delay-dependent and rate-independent robustly exponentially stable in mean square for delays $h(t), r(t)$ satisfying $0<h(t) \leqslant \bar{h}$ and $0<r(t) \leqslant \bar{r}$.

In the following, we will discuss the robust stability for the following uncertain stochastic neural network with time-varying delays

$$
\begin{aligned}
\mathrm{d} x(t)=[ & \left.-A(t) x(t)+W_{0}(t) f(x(t))+W_{1}(t) f(x(t-h(t)))\right] \mathrm{d} t \\
& +\left[C(t) x(t)+D(t) x(t-h(t))+W_{2}(t) f(x(t))+W_{3}(t) f(x(t-h(t)))\right] \mathrm{d} \omega(t),
\end{aligned}
$$

where time-delay $h(t)$ satisfies $0<h(t) \leqslant \bar{h}, \dot{h}(t) \leqslant \mu$. Then, we have the following results.

Theorem 2. Given scalars $\bar{h}>0$, and $\mu$. The equilibrium solution of stochastic neural network (32) is robustly exponentially stable in mean square if there exist matrices $P>0, Q>0, R>0, M_{k}, N_{k}$ and $S_{k}(k=1, \ldots, 6)$ as well as constants $b_{1}>0, b_{2}>0$, $\varepsilon_{1}>0$ and $\varepsilon_{2}>0$, such that the following LMI holds:

$$
\left[\begin{array}{cccc}
\bar{\Xi}_{11} & -N & M H & S H \\
* & -\frac{1}{\bar{h}} R & 0 & 0 \\
* & * & -\varepsilon_{1} I & 0 \\
* & * & * & -\varepsilon_{2} I
\end{array}\right]<0,
$$

where

$$
\bar{\Xi}_{11}=\left(\bar{\phi}_{i j}\right)_{6 \times 6}+\varepsilon_{1} \bar{T}_{1}^{T} \bar{T}_{1}+\varepsilon_{2} \bar{T}_{2}^{T} \bar{T}_{2}
$$

with

$$
\begin{aligned}
& \bar{\phi}_{i j}=\phi_{i j} \quad(i, j=1,2, \ldots, 6, \quad((i, j) \neq(5,5))), \\
& \bar{\phi}_{55}=-b_{1} I+M_{5} W_{0}+W_{0}^{T} M_{5}^{T}+S_{5} W_{2}+W_{2}^{T} S_{5}^{T} .
\end{aligned}
$$

Proof. Consider the Lyapunov-Krasovskii functional for (32) as follows:

$$
V\left(x_{t}, t\right)=x^{T}(t) P x(t)+\int_{t-h(t)}^{t} x^{T}(s) Q x(s) \mathrm{d} s+\int_{-\bar{h}}^{0} \int_{t+\theta}^{t} y^{T}(s) R y(s) \mathrm{d} s \mathrm{~d} \theta,
$$

then (33) follows immediately by following the same lines as proof of Theorem 1 . This completes the proof.

Remark 3. When the differential of $h(t)$ is unknown, by setting $Q=0$ in (34), from Theorem 2, we can obtained the delaydependent and rate-independent robust stability criterion for the system (32).

\section{Numerical examples}

To illustrate the effectiveness of the theory developed in this Letter, we present two numerical examples as follows.

Example 1. Consider the uncertain stochastic neural networks,

$$
\begin{aligned}
\mathrm{d} x(t)= & {\left[-A(t) x(t)+W_{0}(t) f(x(t))+W_{1}(t) f(x(t-h(t)))+E_{1} \int_{t-r(t)}^{t} f(x(s)) \mathrm{d} s\right] \mathrm{d} t } \\
& +\left[C(t) x(t)+D(t) x(t-h(t))+W_{2}(t) f(x(t))+W_{3}(t) f(x(t-h(t)))+E_{2} \int_{t-r(t)}^{t} f(x(s)) \mathrm{d} s\right] \mathrm{d} \omega(t),
\end{aligned}
$$

where

$$
\begin{aligned}
& A=\left[\begin{array}{ll}
4 & 0 \\
0 & 6
\end{array}\right], \quad W_{0}=\left[\begin{array}{cc}
0.2 & -4 \\
0.1 & 0.3
\end{array}\right], \quad W_{1}=\left[\begin{array}{ll}
0.4 & 0.2 \\
0.1 & 0.7
\end{array}\right], \quad W_{2}=\left[\begin{array}{ll}
0.2 & 0.6 \\
0.5 & 0.1
\end{array}\right], \\
& W_{3}=\left[\begin{array}{cc}
0.3 & 0.6 \\
0.2 & -0.1
\end{array}\right], \quad C=\left[\begin{array}{cc}
0.3 & 0 \\
0 & 0.3
\end{array}\right], \quad D=\left[\begin{array}{cc}
0.5 & -0.1 \\
-0.5 & 2.272
\end{array}\right], \quad H=\left[\begin{array}{l}
0.1 \\
0.1
\end{array}\right],
\end{aligned}
$$




$$
E_{1}=E_{2}=I, \quad L=0.2 I, \quad T_{1}=T_{2}=T_{3}=T_{4}=T_{5}=T_{6}=T_{7}=\left[\begin{array}{ll}
1 & 1
\end{array}\right] .
$$

Using Matlab LMI Toolbox, by Theorem 1, for $\mu=0$, it is found that the equilibrium solution of uncertain stochastic neural network (6) is robustly exponentially stable in mean square for any $h(t)$ and $r(t)$ satisfying $0<h(t) \leqslant 2.15,0<r(t) \leqslant 2.15$.

Example 2. Consider the uncertain stochastic time-delay system,

$$
\begin{aligned}
\mathrm{d} x(t)= & {\left[-A(t) x(t)+W_{0}(t) f(x(t))+W_{1}(t) f(x(t-h(t)))\right] \mathrm{d} t } \\
& +\left[C(t) x(t)+D(t) x(t-h(t))+W_{2}(t) f(x(t))+W_{3}(t) f(x(t-h(t)))\right] \mathrm{d} \omega(t),
\end{aligned}
$$

where

$$
\begin{aligned}
& A=\left[\begin{array}{ll}
4 & 0 \\
0 & 5
\end{array}\right], \quad W_{0}=\left[\begin{array}{cc}
0.4 & -0.7 \\
0.1 & 0
\end{array}\right], \quad W_{1}=\left[\begin{array}{cc}
-0.2 & 0.6 \\
0.5 & -0.1
\end{array}\right], \quad W_{2}=W_{3}=\left[\begin{array}{cc}
0.1 & 0 \\
0 & 0.1
\end{array}\right], \\
& C=\left[\begin{array}{cc}
0.5 & 0 \\
0 & 0.5
\end{array}\right], \quad D=\left[\begin{array}{cc}
0 & -0.5 \\
-0.5 & 0
\end{array}\right], \quad H=\left[\begin{array}{c}
0.1 \\
-0.1
\end{array}\right], \quad L=0.5 I, \quad T_{1}=\left[\begin{array}{ll}
0.2 & 0.3
\end{array}\right], \\
& T_{2}=\left[\begin{array}{ll}
0.2 & -0.3
\end{array}\right], \quad T_{3}=\left[\begin{array}{ll}
-0.2 & -0.3
\end{array}\right], \quad T_{4}=T_{5}=T_{6}=T_{7}=\left[\begin{array}{ll}
0.1 & 0.1
\end{array}\right] .
\end{aligned}
$$

For this system, when the differential of $h(t)$ is unknown, applying Theorem 2 in [26], it is found that the equilibrium solution of stochastic neural network (35) is robustly exponentially stable in mean square for any delay $h(t)$ satisfying $0<h(t) \leqslant 0.5730$. However, by Theorem 2 in our Letter, we can conclude that if $0<h(t) \leqslant 0.7056$, system (35) is robustly exponentially stable in mean square.

Specially, when $\Delta C(t)=\Delta D(t)=W_{2}=\Delta W_{2}(t)=W_{3}=\Delta W_{3}(t)=0$, the system became the one in [25]. The uncertain stochastic neural network is globally stable in mean square for the maximum allowed time delay being 0.4109 in [25]. In [26], it is found that this system is robustly exponentially stable in mean square for any delay $h(t)$ satisfying $0<h(t) \leqslant 0.6740$. However, by Theorem 2 in this Letter, it is found that the system is robustly exponentially stable in mean square for any delay $h(t)$ satisfying $0<h(t) \leqslant 0.7795$.

\section{Conclusion}

In this Letter, new robust exponential stability criteria for uncertain stochastic neural with discrete and distributed time-varying delays are presented. By introducing the free-weighting matrix method, less conservative stability results have been proposed. Two numerical examples are used to demonstrate the effectiveness of our results.

\section{Acknowledgements}

The authors are grateful to the anonymous referees for their careful reading and constructive comments and suggestions which helped improving the presentation of the Letter. This work is partially supported by the Natural Science Foundation of China (60674055, 60774047), and the Taishan Scholar programme of Shandong Province.

\section{References}

[1] S. Haykin, Neural Networks: A Comprehensive Foundation, Prentice Hall, New Jersey, 1998.

[2] K. Gopalsamy, X. He, Physica D 76 (1994) 344.

[3] K. Gopalsamy, X. He, IEEE Trans. Neural Networks 5 (1994) 998.

[4] Z. Wang, H. Shu, Y. Liu, D.W.C. Ho, X. Liu, Chaos Solitons Fractals 30 (2006) 886.

[5] S. Xu, J. Lam, D.W.C. Ho, Y. Zou, IEEE Trans. Syst. Man Cybernet. B 35 (2005) 1317.

[6] J. Cao, K. Yuan, H.X. Li, IEEE Trans. Neural Networks 17 (2006) 1646.

[7] Y. He, Q. Wang, W. Zhang, Chaos Solitons Fractals 26 (2005) 1349.

[8] Y. He, G.P. Liu, D. Rees, IEEE Trans. Neural Networks 18 (2007) 310.

[9] J. Cao, IEEE Trans. Circuit Syst.-I 48 (2001) 494.

[10] J. Cao, J. Wang, IEEE Trans. Circuit Syst.-I 52 (2005) 417.

[11] Y. Yang, J. Cao, Physica A 386 (2007) 492.

[12] B. Zhang, S. Xu, Y. Li, Y. Chu, Phys. Lett. A 366 (2007) 69.

[13] S. Xu, J. Lam, D.W.C. Ho, Y. Zou, IEEE Trans. Circuits Syst.-II 52 (2005) 349.

[14] S. Xu, Y. Chu, J. Lu, Phys. Lett. A 352 (2006) 371.

[15] T. Ensari, S. Arik, IEEE Trans. Automat. Control. 50 (2005) 1781.

[16] X. Mao, Stochastic Differential Equations and Their Applications, Horwood, Chichester, 1997.

[17] X. Liao, X. Mao, Stochastic Anal. Appl. 14 (1996) 165.

[18] S. Blythe, X. Mao, X. Liao, J. Franklin Inst. 338 (2001) 481.

[19] L. Wan, J. Sun, Phys. Lett. A 343 (2005) 306.

[20] H. Huang, D.W.C. Ho, J. Lam, IEEE Trans. Circuit Syst.-II 52 (2005) 251. 
[21] X. Lou, B.T. Cui, IEEE Trans. Syst. Man Cybernet. B 37 (2007) 713.

[22] X. Lou, B.T. Cui, J. Math. Anal. Appl. 328 (2007) 316.

[23] C. Li, X. Liao, IEEE Trans. Circuits Syst.-I 53 (2006) 2265.

[24] J. Zhang, P. Shi, J. Qiu, Nonlinear Anal.: Real World Appl. 8 (2007) 1349.

[25] H. Huang, G. Feng, Physica A 381 (2007) 93.

[26] W.-H. Chen, X. Lu, Phys. Lett. A 372 (2008) 1061

[27] R. Rakkiyappan, P. Balasubramaniam, Chaos Solitons Fractals, doi: 10.1016/j.chaos.2007.09.052.

[28] Z. Wang, Y. Liu, K. Fraser, X. Liu, Phys. Lett. A 354 (2006) 288.

[29] Z. Wang, S. Lauria, J. Fang, X. Liu, Chaos Solitons Fractals 32 (2007) 62.

[30] Z. Wang, J. Fang, X. Liu, Chaos Solitons Fractals 36 (2008) 388.

[31] Z. Wang, H. Shu, J. Fang, X. Liu, Nonlinear Anal.: Real World Appl. 7 (2006) 1119.

[32] Y. Liu, Z. Wang, X. Liu, Neurocomput. 70 (2006) 314.

[33] P. Park, C. Chen, Chaos Solitons Fractals 27 (2006) 1011.

[34] Y. Wang, L. Xie, C.E. De Souza, Syst. Control. Lett. 19 (1992) 139. 